\title{
Enhanced Antidepressant-Like Effects of Electroacupuncture Combined with Citalopram in a Rat Model of Depression
}

\author{
Jian Yang, ${ }^{1}$ Yu Pei, ${ }^{1,2}$ Yan-Li Pan, ${ }^{1}$ Jun Jia, ${ }^{3}$ Chen Shi, ${ }^{3}$ Yan Yu, ${ }^{3}$ Jia-Hui Deng, ${ }^{3}$ Bo Li, ${ }^{3}$ \\ Xiao-Li Gong, ${ }^{3}$ Xuan Wang, ${ }^{3}$ Xiao-Min Wang, ${ }^{3}$ and Xin $\mathrm{Ma}^{1}$ \\ ${ }^{1}$ Beijing Anding Hospital, Capital Medical University, 5 Ankang Alley, Beijing 100088, China \\ ${ }^{2}$ School of Arts and Law, Beijing University of Chemical Technology, Beijing 100029, China \\ ${ }^{3}$ Department of Physiology and Neurobiology, Capital Medical University, Key Laboratory for Neurodegenerative Disease of \\ Education Ministry, 10 You An Men, Beijing 100069, China
}

Correspondence should be addressed to Xiao-Min Wang; xmwang@ccmu.edu.cn and Xin Ma; maxinanny@sina.cn

Received 16 February 2013; Revised 13 April 2013; Accepted 22 April 2013

Academic Editor: Vitaly Napadow

Copyright (C) 2013 Jian Yang et al. This is an open access article distributed under the Creative Commons Attribution License, which permits unrestricted use, distribution, and reproduction in any medium, provided the original work is properly cited.

\begin{abstract}
Currently, antidepressants are the dominative treatment for depression, but they have limitations in efficacy and may even produce troublesome side effects. Electroacupuncture (EA) has been reported to have therapeutic benefits in the treatment of depressive disorders. The present study was conducted to determine whether EA could enhance the antidepressant efficacy of a low dose of citalopram (an SSRI antidepressant) in the chronic unpredictable stress-induced depression model rats. Here, we show that a combined treatment with $2 \mathrm{~Hz}$ EA and $5 \mathrm{mg} / \mathrm{kg}$ citalopram for three weeks induces a significant improvement in depressive-like symptoms as detected by sucrose preference test, open field test, and forced swimming test, whereas these effects were not observed with either of the treatments alone. Further investigations revealed that $2 \mathrm{~Hz}$ EA plus $5 \mathrm{mg} / \mathrm{kg}$ citalopram produced a remarkably increased expression of BDNF and its receptor TrkB in the hippocampus compared with those measured in the vehicle group. Our findings suggest that EA combined with a low dose of citalopram could produce greater therapeutic effects, thereby, predictive of a reduction in drug side effects.
\end{abstract}

\section{Introduction}

Depression is a common but serious mental disorder that affects more than $15 \%$ of the population during their lifetime [1]. Currently, antidepressants, especially selective serotonin reuptake inhibitors (SSRIs), are the mainstay in the treatment for depression. Unfortunately, many depressed patients do not respond well to presently available antidepressants and suffer from their severe side effects [2-5]. Therefore, it is necessary to seek complementary and alternative strategies with better efficacy of antidepressants and fewer side effects.

Acupuncture is a traditional complementary and alternative medicine approach that involves inserting fine needles into specific points to restore proper energy flow inside the body [6]. Electroacupuncture (EA) is a modification of acupuncture in which the needles inserted are attached with electrodes to deliver a pulsed electrical current. Numerous studies have demonstrated that acupuncture or EA treatment could alleviate depressive symptoms with very few side effects [7-10]. However, some researchers believe that there is a lack of sufficient evidence for supporting a beneficial effect from them $[11,12]$. Although their utility in treating depression remains controversial, either of them may be considered as an adjunct to standard therapy $[13,14]$.

Brain-derived neurotrophic factor (BDNF), a member of the neurotrophin family, plays critical roles in cell differentiation, neuronal survival, migration, and synaptic plasticity [15-17] and has been implicated in the pathophysiology of depression [18-21]. Postmortem studies have shown that hippocampal BDNF levels are decreased in depressed patients and increased in patients receiving antidepressant treatment [22-24]. Animal studies have demonstrated that various types of acute (e.g., immobilization and footshock) and chronic (e.g., chronic unpredictable stress (CUS) and chronic restrain) stress paradigms reduce BDNF expression in the hippocampus, and this reduction can be reversed by chronic 
TABLE 1: Schedule of chronic mild stress procedures.

\begin{tabular}{lcc}
\hline Day & Stressors & Time \\
\hline 1 & 10 h crowded cage $(5-6$ rats per cage $)$ & a.m. to p.m. \\
2 & 15 min forced swim $\left(22^{\circ} \mathrm{C}\right)$ & p.m. \\
& 15 h wet bedding & \\
3 & 4 h restraint & a.m. \\
4 & 24 h food deprivation & p.m. \\
& 5 min cold swim $\left(4^{\circ} \mathrm{C}\right)$ & \\
5 & 4 h restraint & a.m. \\
& 24 h water deprivation & \\
6 & (including 6 h empty water bottle $)$ & a.m. \\
& 2 h cold stress $\left(15^{\circ} \mathrm{C}\right)$ & p.m. \\
7 & 4 h restraint & a.m. \\
& 2 h restraint & p.m. \\
\hline
\end{tabular}

antidepressant treatment [25-28]. Furthermore, several lines of evidence suggest that acupuncture or EA can upregulate hippocampal BDNF expression in normal and depression model rats [29-31].

Considering all of the aforementioned, the present study was designed to determine whether EA intervention combined with citalopram (a widely used SSRI), at a lower dose than what is required for monotherapy, could produce greater therapeutic effects in the CUS rats compared with EA or citalopram treatment alone. Changes in BDNF and its major receptor tropomyosin-related kinase receptor $\mathrm{B}$ (TrkB) were also evaluated.

\section{Materials and Methods}

2.1. Animals. Adult Sprague-Dawley (SD) rats (male, 200$220 \mathrm{~g}$ ) were obtained from the laboratory animal center, Capital Medical University. Animals were maintained in a standard $12 \mathrm{~h}$ light/dark cycle in cages with ad libitum access to food and water and were allowed to acclimate to the environment for 7 days. All experimental procedures were approved by the Ethics Committee on Animal Care and Usage of Capital Medical University. Every effort was made to minimize animal suffering.

2.2. Experimental Design and Animal Groups. Rats in the control group received no CUS during the whole experiment. In the model group, rats were exposed to CUS for 7 weeks. From the beginning of the 5th week, model rats were randomly grouped and subjected to different experiments. In the first experiment, rats were divided into five groups and each group had 8-9 rats. Normal rats were intraperitoneally (i.p.) injected with saline, and model rats were, respectively, injected (i.p.) with saline and 5, 10, and $20 \mathrm{mg} / \mathrm{kg}$ citalopram once a day for 3 weeks. In the second experiment, there are four groups and each group had 8 rats. Normal rats received no treatment, while model rats were subjected to EA stimulation for 3 weeks and further divided into three groups: vehicle (received CUS only), $2 \mathrm{~Hz}$ EA, and $100 \mathrm{~Hz}$ EA. In the third experiment, model rats were administered with combined treatment with EA and citalopram for 3 weeks. In

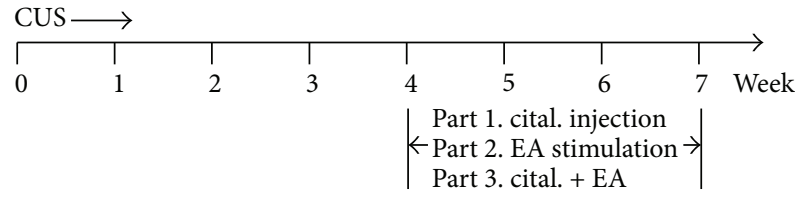

FIGURE 1: Schematic representation of the experimental procedure.

summary, there were five groups and each group had 8-9 rats: (1) vehicle (received CUS only); (2) $2 \mathrm{~Hz}$ EA group; (3) Cital 5 group; (4) Cital 5 plus $2 \mathrm{~Hz}$ EA group; (5) Cital 10 group. The experimental procedure is shown in Figure 1.

2.3. CUS Procedure. The CUS protocol was modified from the procedures described by Katz [32] and Willner et al. [33]. It consisted of a variety of sequential stressors applied randomly every day for 7 weeks (Table 1). Rats in the nonstress group were housed in groups of three, unless when they were subjected to sucrose preference test. Those exposed to CUS procedure were housed alone, unless when they were subjected to high-density housing.

2.4. Sucrose Preference Test. The sucrose preference test was performed as previously described with minor modification $[34,35]$. This test was carried out before CUS and at the end of each week. Rats were kept individually in separate cages and habituated to two needleless syringes (resp., filled with plain water and $1 \%$ sucrose solution) $8 \mathrm{~h}$ per day for 2 days. Followed by $12 \mathrm{~h}$ period of food and water deprivation, the rats were exposed to the two syringes for $30 \mathrm{~min}$. After an interval of $1 \mathrm{~h}$, the positions of the syringes were exchanged, and the rats were tested for $30 \mathrm{~min}$ again. The volume of sucrose solution and water consumption during the total $1 \mathrm{~h}$ test was recorded. Sucrose preference was expressed as a ratio of the volume of sucrose solution consumption to the volume of total fluid intake.

2.5. Open Field Test (OFT). Locomotor activity was monitored automatically with infrared beams (each beam space $2.5 \mathrm{~cm}$ ) in a black chamber before CUS and at the end of 4 th and 7 th weeks during the CUS procedure (TruScan 2.0 Instruments, Columbus, OH.). Tests were conducted between 9 a.m. and 11 a.m.. Each rat was placed in a corner of the chamber and was allowed to explore freely for $5 \mathrm{~min}$. At the end of each test, the chamber was cleaned with $70 \%$ ethanol solution to remove any olfactory cues. Locomotor activity was defined as horizontal (floor plane, FP) and vertical (vertical plane, VP) movement distances and the number of entries into the arena-center (defined as more than 2.5-beam spaces away from the arena walls). Movement distances provide information on general activity. Number of center entries probably reflects anxiety-like behavior, with more "anxious" rats entering fewer times into the arenacenter. The data were recorded and analyzed using a DigiScan analyzer and software (TruScan 2.0, Columbus).

2.6. Forced Swimming Test (FST). FST was performed at the end of 4 th and 7 th weeks during the CUS procedure. This 
test consists of a 15 min pretest swim and a 5 min test swim on the following day [36]. Rats were forced to swim in a glass cylinder (diameter $26 \mathrm{~cm}$ and height $60 \mathrm{~cm}$ ) containing $35 \mathrm{~cm}$ depth of water at a temperature of $25^{\circ} \mathrm{C}$. Water was changed after each test. Behavior was video-recorded using SMART video-tracking system (Panlab, Spain). Three types of behavior were analyzed: immobility, swimming, and climbing [37]. Immobility was defined as floating with no active activity other than those necessary to keep head above the water. Swimming was defined as active movements throughout the cylinder, including crossing into another quadrant. Climbing was defined as upward-directed movements of the forepaws against the cylinder walls.

2.7. Citalopram Treatment. Citalopram hydrobromide (Sigma-Aldrich, MO, USA) was dissolved in $0.9 \%$ physiological saline immediately before use and administered intraperitoneally daily from the 5 th week to the 7 th week during the CUS procedure. Control rats received saline as a vehicle injection.

2.8. EA Treatment. EA stimulation was administered from the 5th week following the CUS procedure. Two stainless steel needles of $0.25 \mathrm{~mm}$ in diameter were inserted at a depth of $5 \mathrm{~mm}$ into the acupoints of BAIHUI (GV 20, at the midpoint between the auricular apices) and Yintang (EX-HN 3, at the midpoint between the eyebrows). The bidirectional squarewave $(0.2 \mathrm{~ms})$ electrical pulse from a medical EA apparatus (HANS LY-257, Beijing) was administered with frequency 2 or $100 \mathrm{~Hz}$ for a total of $30 \mathrm{~min}$ each day, 6 days per week. The intensity of the stimulation was increased stepwise from 1 to $3 \mathrm{~mA}$. During EA stimulation, the rats were kept under awake and unrestrained conditions in individual cages.

2.9. BDNF Protein Detection. Four to five rats in each group were killed by decapitation one day after behavioral measurements. Hippocampal tissue was dissected for protein assays and trunk blood was collected for the determination of serum BDNF levels. Samples were stored at $-80^{\circ} \mathrm{C}$ until assay. Each frozen hippocampus was homogenized and lysed with RIPA buffer containing protease inhibitor cocktail (SigmaAldrich). The total protein concentration was determined using BCA protein assay kit (Pierce, Rockford, IL). For Western blot analysis, equal amounts of proteins were separated by $12 \%$ SDS-PAGE and transferred to PVDF (polyvinyldifluoride) membranes (Millipore). After being blocked with $5 \%$ nonfat-dried milk for $1 \mathrm{~h}$, the membranes were incubated overnight at $4^{\circ} \mathrm{C}$ with rabbit polyclonal antibody to BDNF (1:300, Santa Cruz), rabbit polyclonal antibody to TrkB ( $1: 1000$, Millipore), and mouse monoclonal antibody to $\beta$ actin (1:5000, Sigma-Aldrich). Then the membranes were incubated with IRDye 800 conjugated secondary antibodies (Rockland Immunochemicals). Signals were visualized by the Odyssey infrared double-fluorescence imaging system (American Company LI-COR Biosciences, Lincoln, NE, USA). For enzyme-linked immunoassay (ELISA), hippocampal and serum BDNF levels were analyzed using a Chemokine BDNF ELISA kit according to the manufacturer's protocol
(Millipore, Billerica, MA). The optical density was measured at $450 \mathrm{~nm}$ using an ELISA reader (Bio-Rad Laboratories Ltd, CA). ELISA results were expressed as ng per $\mathrm{mL}$ serum and pg per mg protein. All samples were assayed in duplicate.

2.10. Statistical Analysis. Data were presented as means \pm SEM. Statistical significance was assessed with the Student's $t$-test or one-way analysis of variance (ANOVA) followed by Newman-Keuls as post hoc multiple comparisons test using Prism 5.0 software (GraphPad Software). $P$ value less than 0.05 was considered to be statistically significant.

\section{Results}

3.1. Chronic Unpredictable Stress Model. Rats subjected to CUS exhibited significantly lower body weight compared with nonstressed controls from the first week after the commencement of CUS procedure (Week 1: control: $272.0 \pm$ 4.028 g, CUS: $259.0 \pm 2.152$ g; Week 2: control: $290.6 \pm 3.461 \mathrm{~g}$, CUS: $261.4 \pm 2.824 \mathrm{~g}$; Week 3: control: $307.5 \pm 3.090 \mathrm{~g}$, CUS: $261.6 \pm 1.946 \mathrm{~g}$; Week 4: control: $331.3 \pm 2.916 \mathrm{~g}$, CUS: $266.4 \pm 2.386$ g.) (Figure 2(a)). Decreased sucrose preference is considered as a symptom resembling anhedonia, which is a core clinical feature of depression in humans [38, 39]. After 4 weeks of CUS, there was a significant decrease in sucrose preference in the model rats as compared with the controls, while the total fluid intake was not affected (Figures 2(b) and 2(c)). In the FST, the model rats demonstrated a significant increase in immobility time, accompanied by remarkably decreased swimming and climbing time (Figure 2(d)). Moreover, when exposed to the OFT, those rats showed significant decreases in the horizontal and vertical movement distances and fewer times into the arena-center (Figures 2(e)-2(g)). Taken together, these results indicate that the 4 weeks of CUS procedure is able to induce depressive-like symptoms (i.e., anhedonia, behavioral despair, and reduced locomotor activity) in the model rats.

3.2. Chronic Administration of Citalopram Ameliorates Depressive-Like Behavior. Model rats were intraperitoneally injected with different doses of citalopram from the 5 th week of CUS procedure. After 3 weeks of administration, both 10 and $20 \mathrm{mg} / \mathrm{kg}$ citalopram produced significantly increased sucrose preference, without affecting the total fluid intake (Figures 3(a) and 3(b)). In addition, they induced dramatic increases in horizontal and vertical movement distances (Figures 3(d) and 3(e)) and center entries (Figure 3(f)) in the OFT. However, these effects were not detected by treatment with $5 \mathrm{mg} / \mathrm{kg}$ citalopram. Moreover, none of these three doses of citalopram exerted any notable effect in the FST (Figure 3(c)). Thus, $5 \mathrm{mg} / \mathrm{kg}$ citalopram was considered ineffective and would be chosen to be combined with EA for further investigation.

3.3. EA Treatment Alone Only Improves Locomotor Activity of CUS Rats. To assess whether EA could improve depressivelike behavior, model rats were administered with frequency 2 or $100 \mathrm{~Hz}$ EA stimulation from the 5th week of CUS 


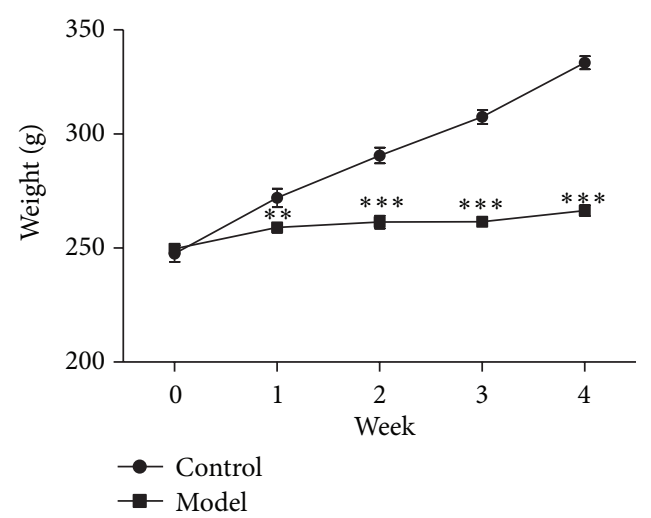

(a)

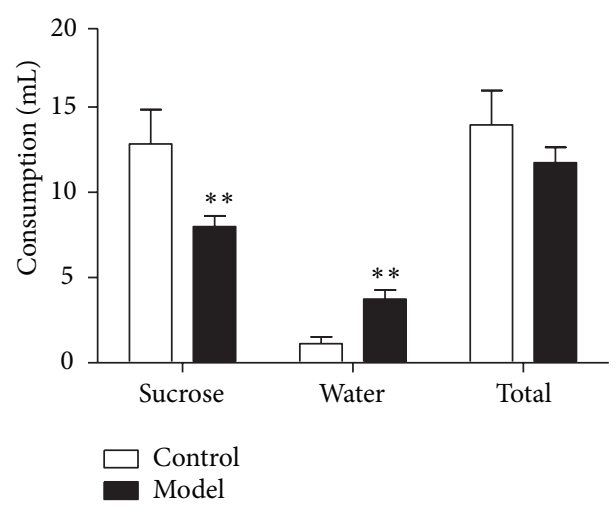

(c)

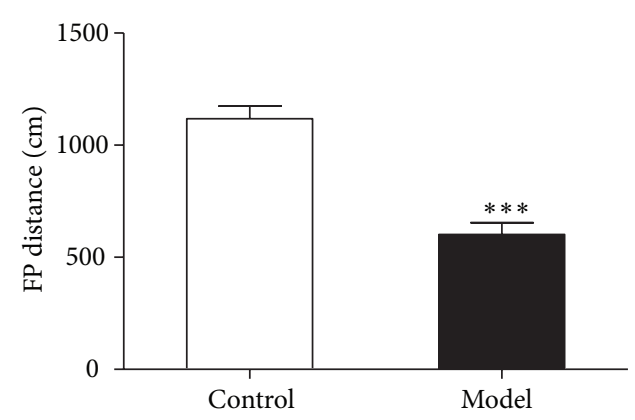

(e)

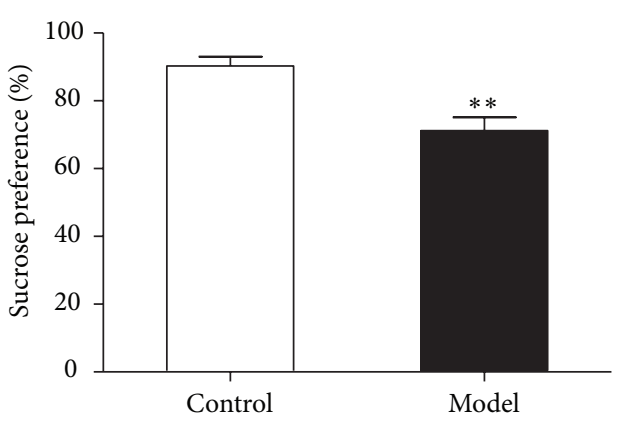

(b)

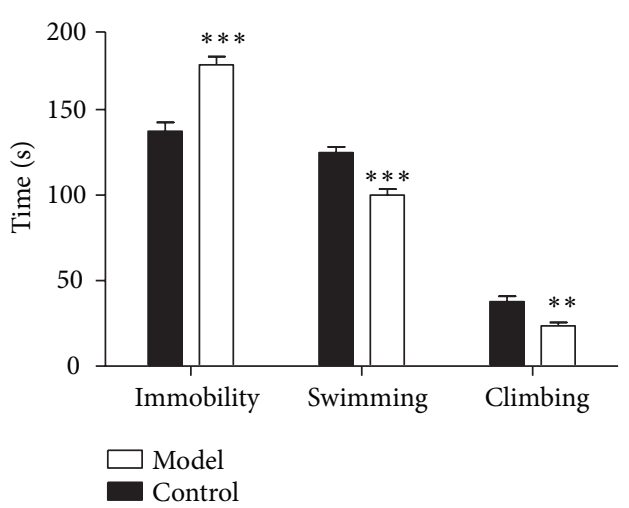

(d)

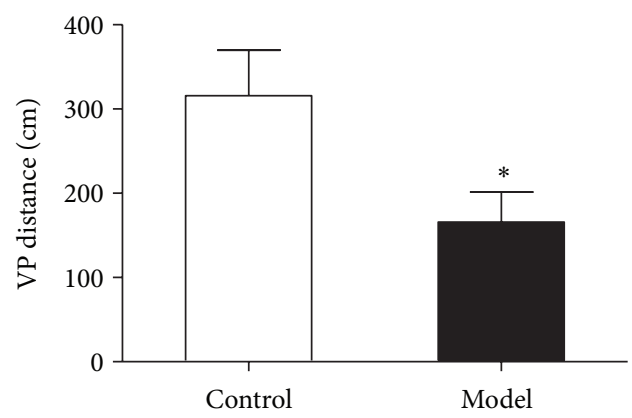

(f)

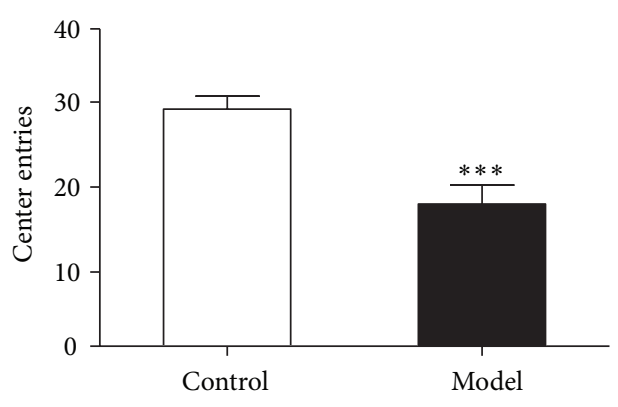

(g)

FIGURE 2: Rats show depressive-like symptoms after 4 weeks of CUS procedure. (a) Body weight. (b) Sucrose preference expressed as a ratio of the volume of sucrose solution consumption to the volume of total fluid intake. (c) The volume of consumption of sucrose solution, water, and total fluid. (d) FST. Data (expressed in seconds) are presented as time spent in immobility, climbing, and swimming. (e)-(g) OFT. Data (expressed in centimeter) are presented as movement distances in the floor plane (FP) (e) and vertical plane (VP) (f) and the number of entries into the arena-center $(\mathrm{g}) . n=11-15$ per group. Data represent mean $\pm \mathrm{SEM},{ }^{*} P<0.05,{ }^{* *} P<0.01$, and ${ }^{* * *} P<0.001$ versus the control group. 

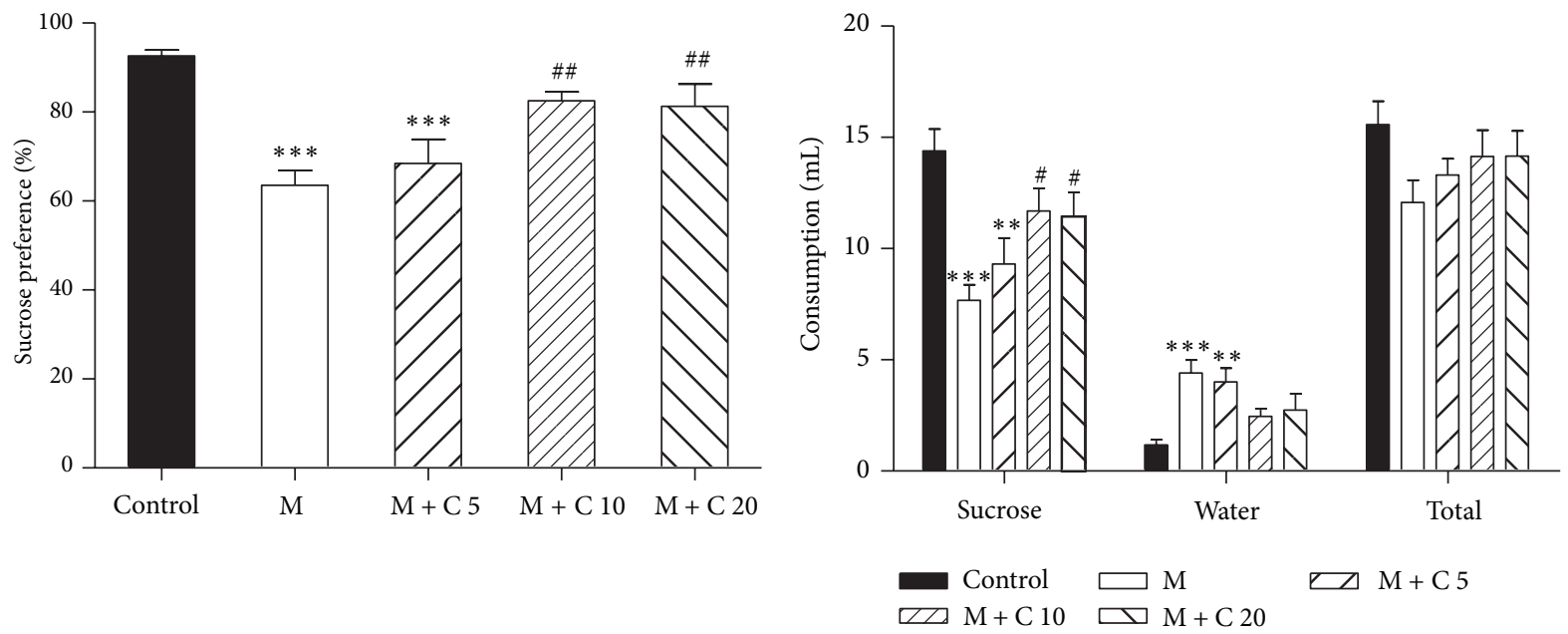

(a)

(b)
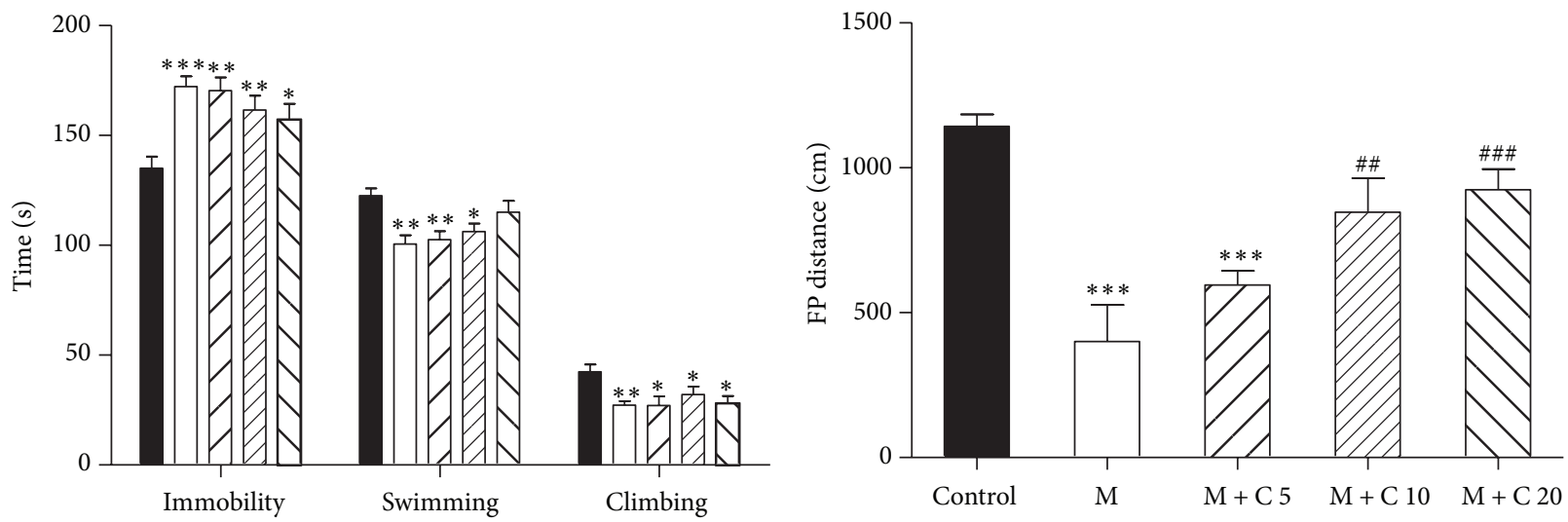

$\begin{array}{ll}\text { Control } \\ \mathrm{M}+\mathrm{C} 10 \\ \mathrm{M}\end{array} \mathrm{M}+\mathrm{C} 20 \square \mathrm{M}+\mathrm{C} 5$

(c)

(d)

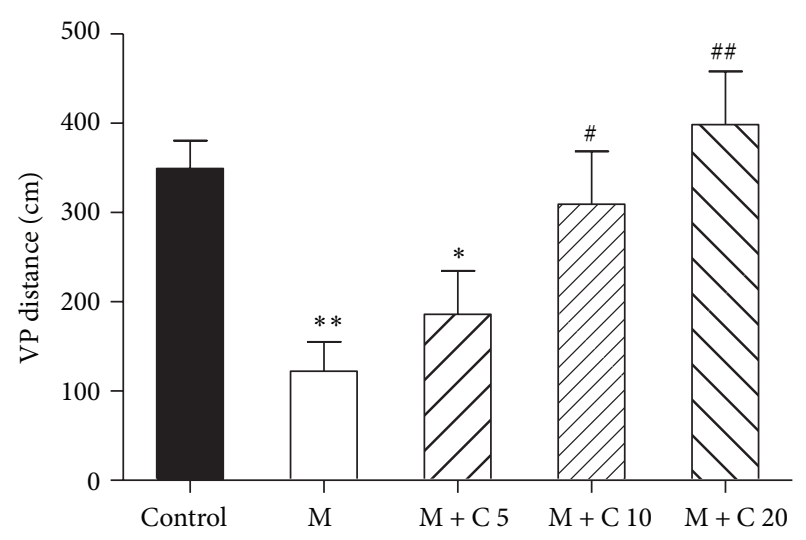

(e)

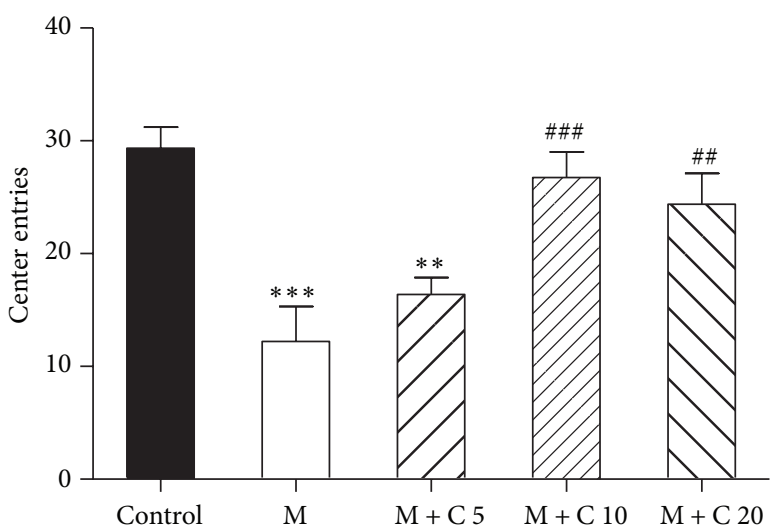

(f)

FIGURE 3: Effects of 3 weeks of treatment with citalopram on CUS-induced depressive-like behavior. (a) Citalopram significantly increased sucrose preference at 10 and $20 \mathrm{mg} / \mathrm{kg}$ but not $5 \mathrm{mg} / \mathrm{kg}$. (b) Citalopram remarkably increased sucrose intake without affecting the total fluid intake. (c) Citalopram at different doses $(5,10$, and $20 \mathrm{mg} / \mathrm{kg}$ ) had no effect on immobility, climbing, and swimming in the FST. (d)-(f) In the OFT, citalopram significantly increased horizontal (d) and vertical (e) movement distances and center entries (f) at 10 and $20 \mathrm{mg} / \mathrm{kg}$ but not $5 \mathrm{mg} / \mathrm{kg} . n=8-9$ per group. Data represent mean $\pm \mathrm{SEM},{ }^{*} P<0.05$, and ${ }^{* *} P<0.01$ versus the vehicle-treated group. 
procedure. After 3 weeks of treatment, neither 2 nor $100 \mathrm{~Hz}$ EA stimulation was able to improve anhedonia and behavioral despair as detected by sucrose preference test and FST (Figures 4(a)-4(c)). By contrast, both 2 and $100 \mathrm{~Hz}$ EA stimulation significantly increased vertical movement distance and center entries in the OFT, but only $2 \mathrm{~Hz}$ EA stimulation demonstrated a significant effect on the horizontal movement distance (Figures 4(d)-4(f)). Thus, we next explored the joint effects of $2 \mathrm{~Hz}$ EA and $5 \mathrm{mg} / \mathrm{kg}$ citalopram in the subsequent experiment.

\subsection{Enhanced Antidepressant Effects of Combined Treatment} with EA and Citalopram. To evaluate whether EA combined with citalopram has an additive or synergistic antidepressant effect, combined treatment with $2 \mathrm{~Hz}$ EA and a low dose of citalopram was administered from the 5 th week of CUS procedure. After 3 weeks, $2 \mathrm{~Hz}$ EA plus $5 \mathrm{mg} / \mathrm{kg}$ citalopram led to substantial increases in sucrose preference (Figures 5(b) and 5(c)), horizontal/vertical movement distances (Figures 5(e) and 5(f)), and center entries (Figure 5(g)), demonstrating similar effects to $10 \mathrm{mg} / \mathrm{kg}$ citalopram. Furthermore, $2 \mathrm{~Hz}$ EA plus $5 \mathrm{mg} / \mathrm{kg}$ citalopram induced a significant reduction in immobility time, accompanied by an increased climbing time in the FST, whereas these effects were not detected by treatment with $10 \mathrm{mg} / \mathrm{kg}$ citalopram (Figure 5(d)). However, there was no remarkable difference in body weight among all the groups (Figure 5(a)). These findings suggest that $2 \mathrm{~Hz}$ EA plus $5 \mathrm{mg} / \mathrm{kg}$ citalopram could exert better antidepressant effects in comparison with either treatment alone.

\subsection{Combined Treatment with EA and Citalopram Induces a} Higher Expression of BDNF in the Hippocampus. To explore the possible mechanisms involved in the joint effects of EA and citalopram, the rats were sacrificed one day after behavioral tests. Results from ELISA demonstrated that hippocampal BDNF protein levels were significantly decreased after 4 weeks of CUS procedure (Figure 6(a)). However, 3 weeks of treatment with $2 \mathrm{~Hz}$ EA plus $5 \mathrm{mg} / \mathrm{kg}$ citalopram, as well as $10 \mathrm{mg} / \mathrm{kg}$ citalopram, led to a remarkable increase in BDNF expression in the hippocampus (Figure 6(b)), but not in the prefrontal cortex or serum (data not shown). Western blot analysis also showed that protein levels of both mature BDNF (mBDNF) and BDNF precursor (proBDNF) significantly increased in $2 \mathrm{~Hz}$ EA plus $5 \mathrm{mg} / \mathrm{kg}$ citalopram group, similar to the observations in $10 \mathrm{mg} / \mathrm{kg}$ citalopram group. Moreover, both of these groups revealed a dramatic increase in the expression of TrkB compared with that measured in the vehicle group (Figure 6(c)). These data indicate that EA combined with citalopram could prevent CUS-induced decrease in BDNF signaling in the hippocampus.

\section{Discussion}

The present study aimed at exploring the therapeutic potentiality of coadministration of EA and an antidepressant by biochemical and behavioral approaches using an animal model predictive of antidepressant-like activity. Here, we demonstrated that the combined treatment with $2 \mathrm{~Hz}$ EA and a low dose of citalopram could prevent CUS-induced decrease in hippocampal BDNF signaling and exert better antidepressant effects in the CUS model rats than either treatment alone.

CUS model, which mimics socioenvironmental stressors in everyday life, is one of the most extensively used animal models of depression [40]. Rats subjected to the CUS paradigm for several weeks can exhibit almost all demonstrable depressive symptoms. However, this model is difficult to replicate, because different CUS schedules, including types of stressors, animal strains, and nutritive status, can result in inconsistent findings $[39,41]$. It has been reported that chronic restraint stress can induce significant downregulation of BDNF in the hippocampus [27, 42]. Accordingly, restraints were used frequently in our CUS paradigm. Consistent with previous studies, we have demonstrated that rats subjected to CUS for 4 weeks exhibit a significantly decreased sucrose preference, accompanied by other behavioral changes such as increased immobility time and decreased locomotor activity.

FST is widely used as a screening procedure for antidepressants [37, 43]. In this test, animals display "despair" behavior (immobility) and active behaviors (swimming and climbing) (14). It has been demonstrated that different SSRIs may exert different effects on immobility time [44, 45]. In our experiment, citalopram was demonstrated inactive after chronic administration in the FST, consistent with several previous researches [46, 47]. On the contrary, many other studies have shown positive effects of acute administration of citalopram on immobility and swimming time in acute stress models [48-50]. The missing observation of significant changes in our experiment might be attributed to two main causes. One is the rat strain. It has been shown that SD rats respond to various antidepressants not so well as WistarKyoto rats in the FST [44]. The other is the type of stressors. Repeated swimming stress may depress the sensitivity of model rats to FST. Also, it has been reported that food restriction with modest weight reduction can attenuate the behavioral effects of SSRIs [51]. Even so, we demonstrated that high doses (10 and $20 \mathrm{mg} / \mathrm{kg}$ ) of citalopram were able to result in significantly increased sucrose preference and improved locomotor activity in the model rats as previously reported $[52,53]$.

Acupuncture has been applied to treat depressive disorders with a long clinical history, but a recent Cochrane review identified that no consistent benefit was noted with any form of acupuncture (manual acupuncture, EA, or laser acupuncture) in depressed patients $[6,14]$. In the present study, we found that sucrose preference and immobility time in the model rats were not significantly affected by EA treatment, even though there was a tendency of increase or reduction after 3 weeks of stimulation. These results are in agreement with several previous studies that were also performed on the CUS model rats [54-56]. Liu and his colleagues demonstrated that 3 weeks of EA stimulation produced a significant increase in the number of crossing in the OFT and a nonsignificant increase in the sucrose intake [55]. Another study reported by $\mathrm{Yu}$ et al. showed that sucrose preference and immobility time in the depressive rats were not significantly changed by 6 weeks of treatment with EA alone [56]. Because frequency 

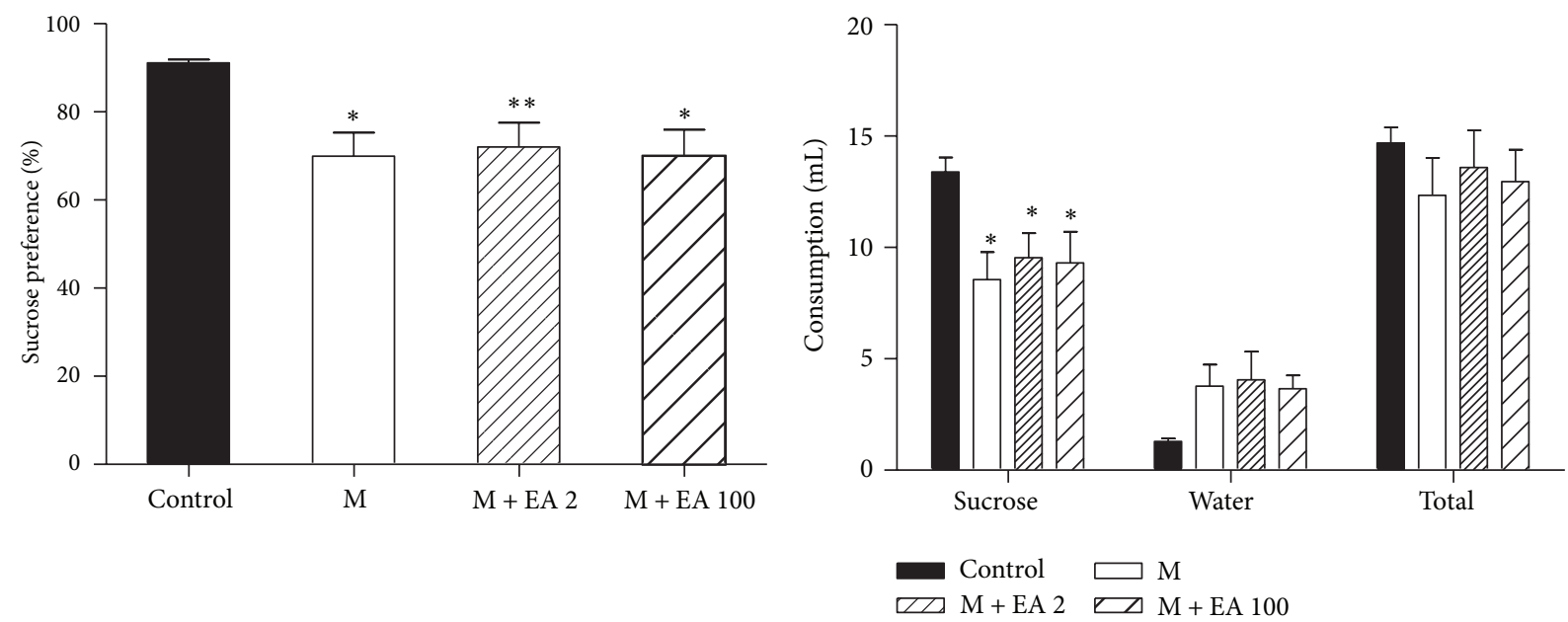

(a)

(b)
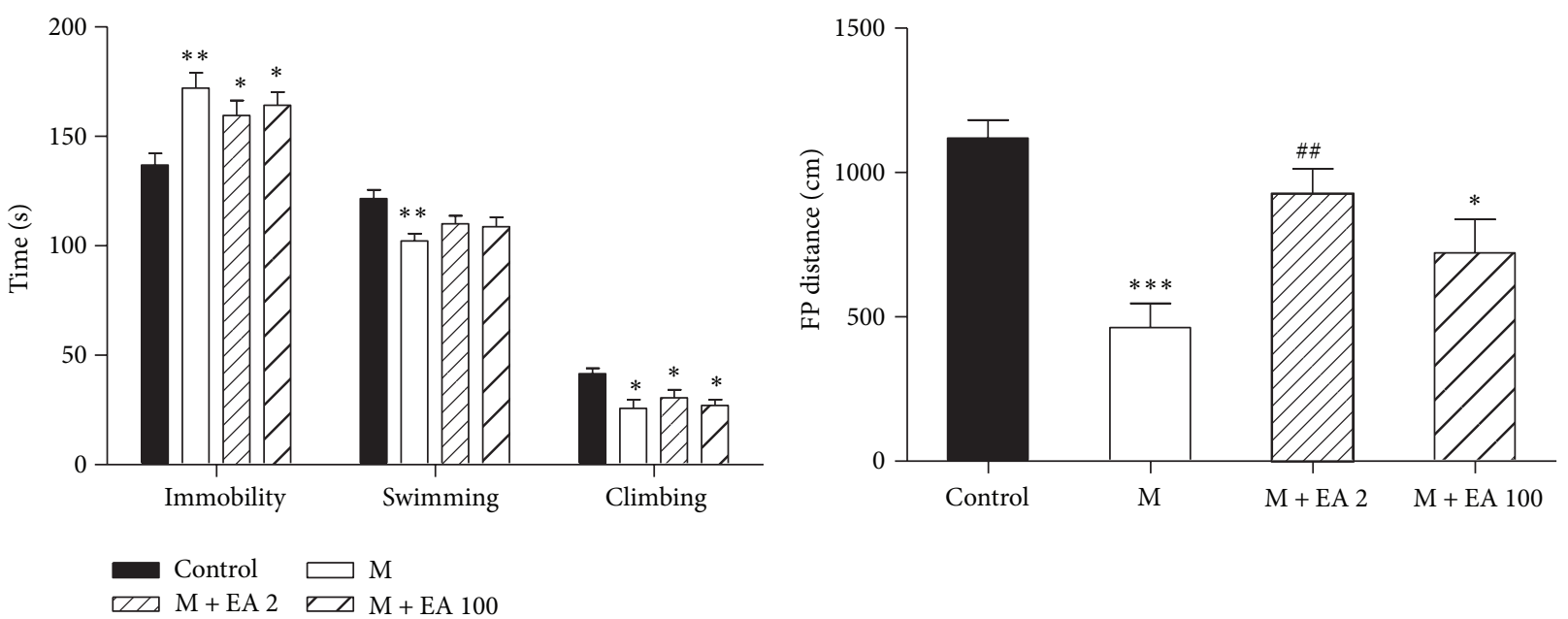

(c)

(d)
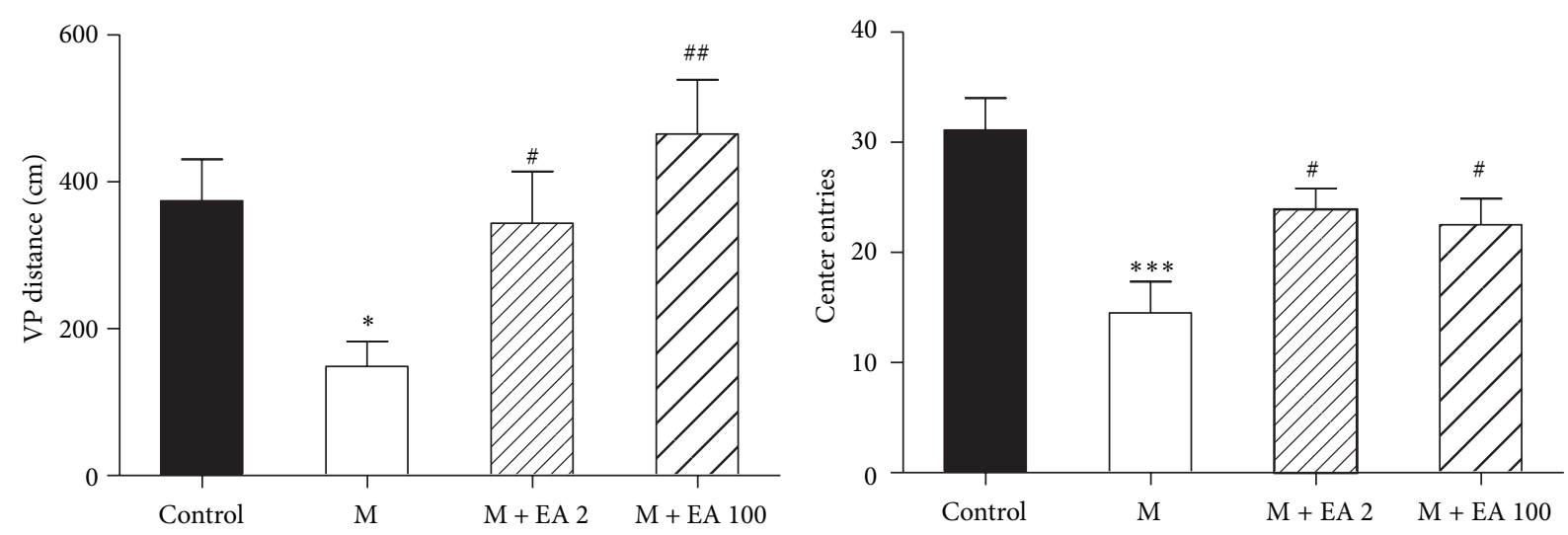

(e)

(f)

FIGURE 4: Effects of 3 weeks of EA stimulation on CUS-induced depressive-like behavior. (a)-(b) $2 \mathrm{~Hz}$ or $100 \mathrm{~Hz}$ EA stimulation had no effect on sucrose preference and sucrose intake. (c) Neither $2 \mathrm{~Hz}$ nor $100 \mathrm{~Hz}$ EA had any effect on immobility, climbing, and swimming in the FST. (d)-(f) In the OFT, $2 \mathrm{~Hz}$ EA induced significantly increased horizontal (d) and vertical (e) movement distances and center entries (f), while $100 \mathrm{~Hz}$ EA demonstrated no effect on the horizontal movement distance. $n=8$ per group. Data represent mean \pm SEM, ${ }^{*} P<0.05$, and ${ }^{* *} P<0.01$ versus the vehicle-treated group. 

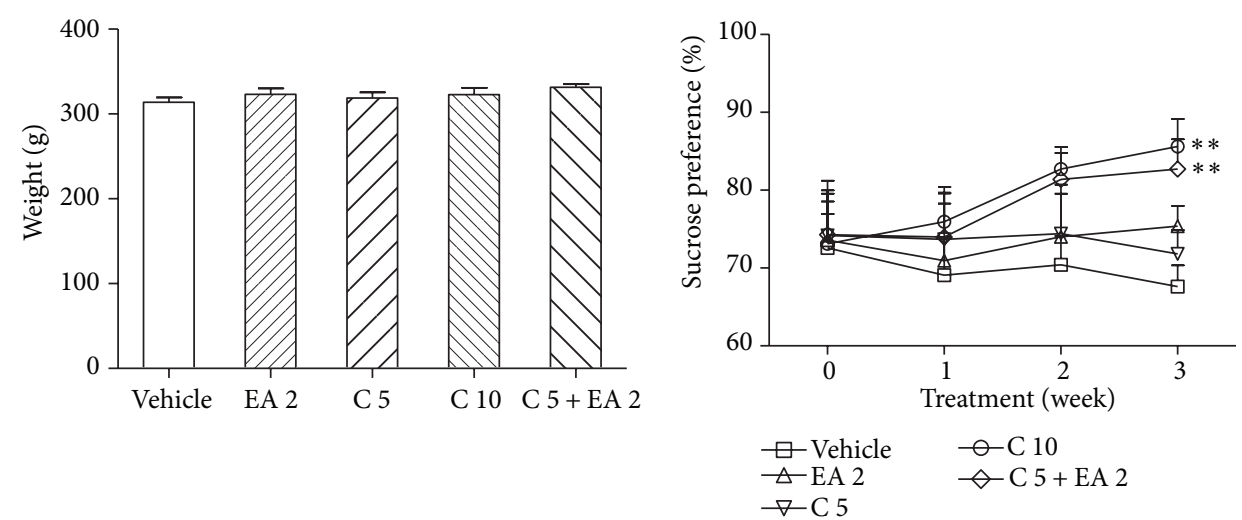

(a)
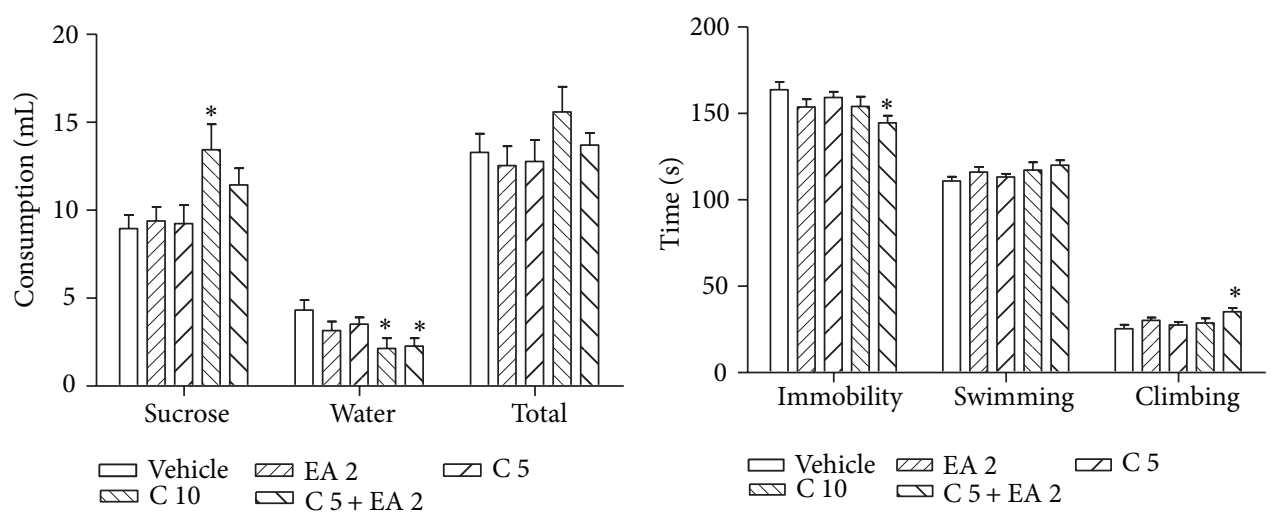

(c)

(d)
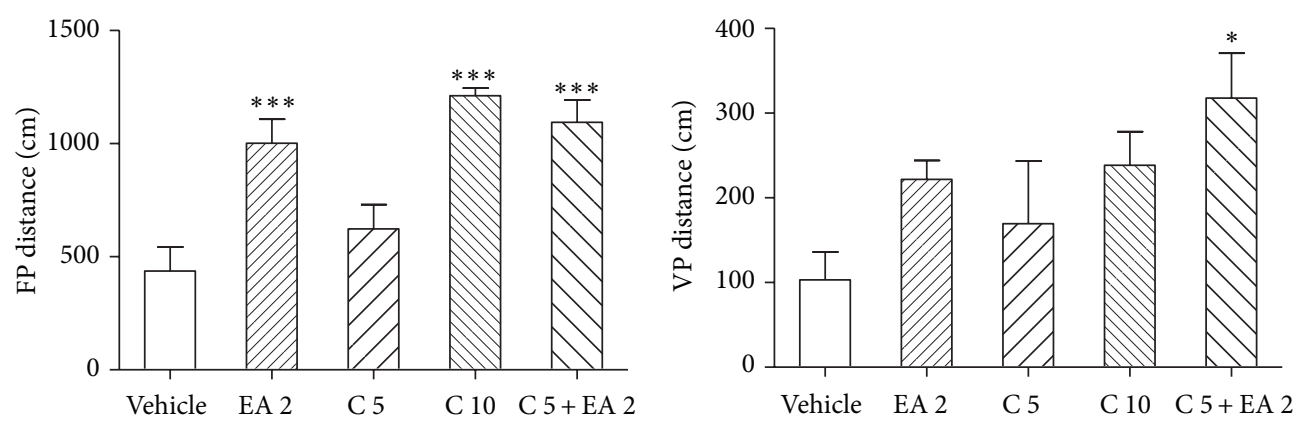

(e)

(f)

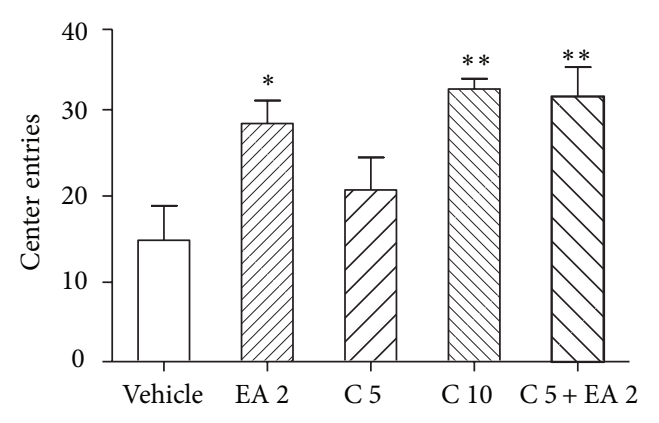

(g)

FIGURE 5: Effects of 3 weeks of combined treatment with EA and citalopram on CUS-induced depressive-like behavior. (a) Body weight. (b)(c) $2 \mathrm{~Hz}$ EA plus $5 \mathrm{mg} / \mathrm{kg}$ citalopram as well as $10 \mathrm{mg} / \mathrm{kg}$ citalopram produced a substantial increase in sucrose preference without affecting the total fluid intake. (d) $2 \mathrm{~Hz}$ EA plus $5 \mathrm{mg} / \mathrm{kg}$ citalopram induced significantly decreased immobility time and increased climbing time in the FST. (e)-(g) $2 \mathrm{~Hz}$ EA plus $5 \mathrm{mg} / \mathrm{kg}$ citalopram led to significantly increased horizontal (e) and vertical (f) movement distances and center entries (g). $n=8$-9 per group. Data represent mean \pm SEM, ${ }^{*} P<0.05,{ }^{* *} P<0.01$, and ${ }^{* * *} P<0.001$ versus the vehicle-treated group. 


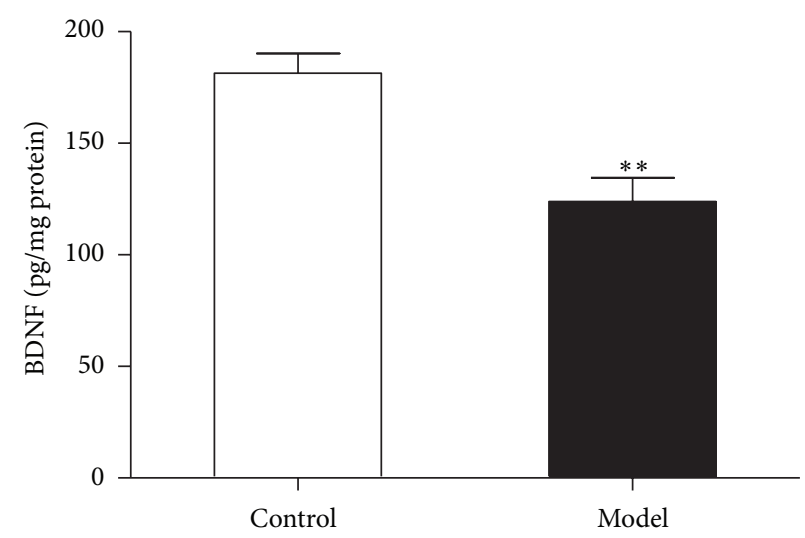

(a)

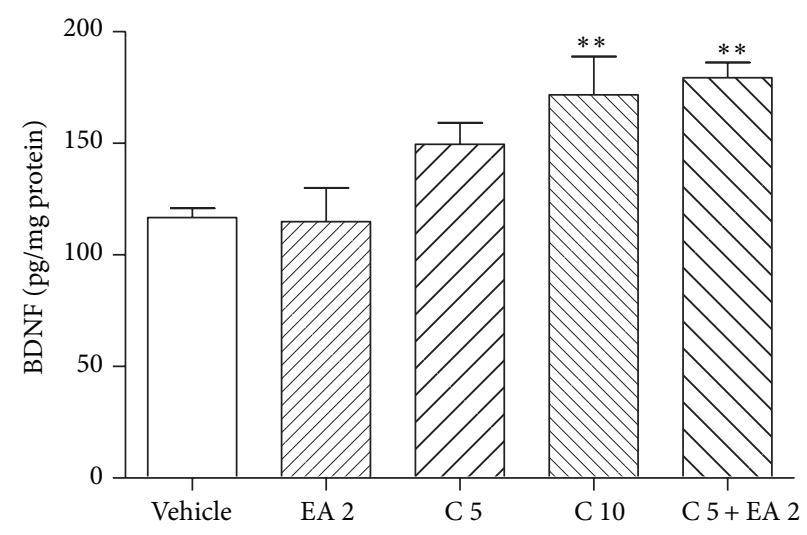

(b)

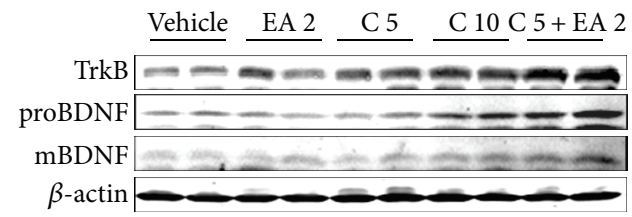

(c)

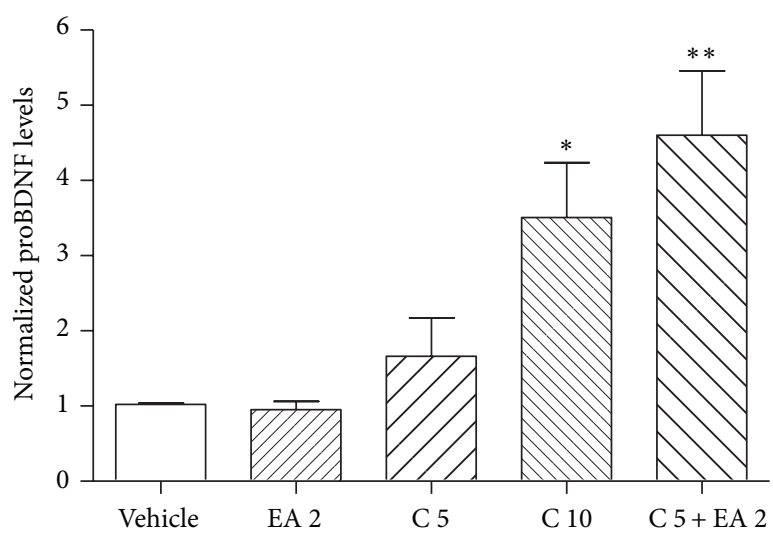

(e)

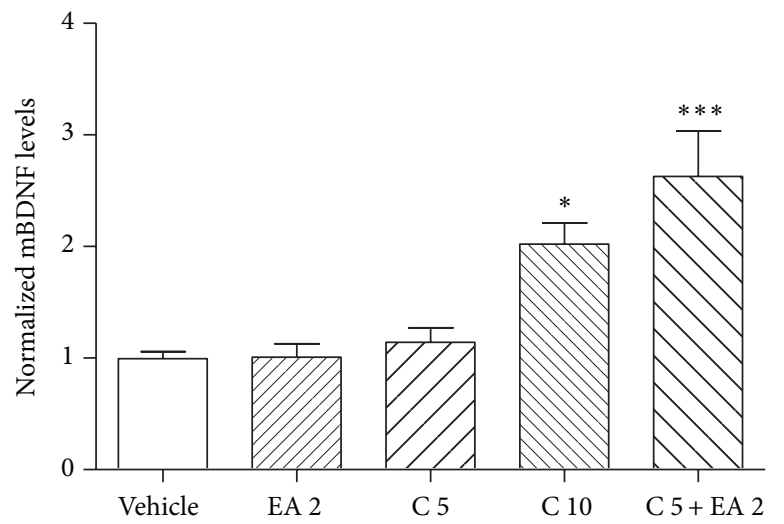

(d)

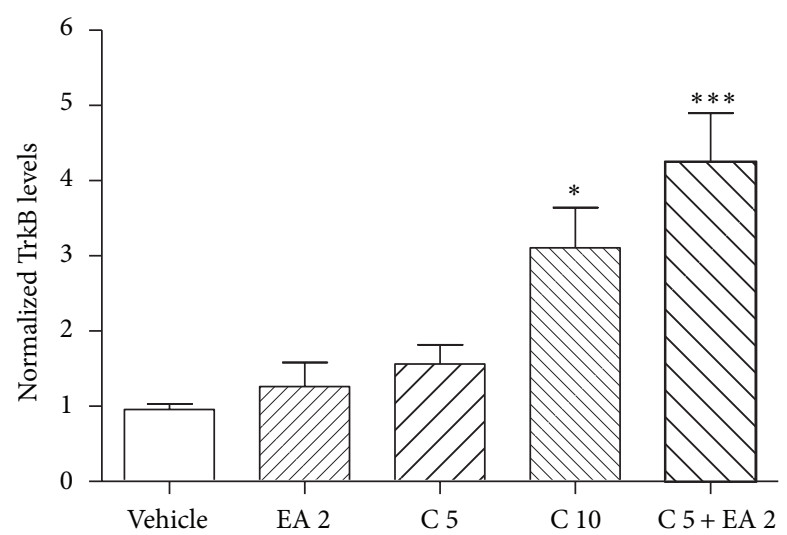

(f)

FIGURE 6: CUS-induced downregulation of BDNF expression was reversed by 3 weeks of combined treatment with EA and citalopram. (a) Hippocampal BDNF protein levels were significantly decreased after 4 weeks of CUS procedure. (b) ELISA results showed that $2 \mathrm{~Hz}$ EA plus $5 \mathrm{mg} / \mathrm{kg}$ citalopram as well as $10 \mathrm{mg} / \mathrm{kg}$ citalopram induced remarkably increased BDNF protein levels in the hippocampus. (c) Western blot analysis of hippocampal mBDNF, proBDNF, and TrkB protein levels. $\beta$-actin was used as an internal control. (d)-(f) Quantitative analysis of mBDNF, proBDNF, and TrkB protein levels in (b). $n=4-5$ per group. Data represent mean \pm SEM, ${ }^{*} P<0.05,{ }^{* *} P<0.01$, and ${ }^{* * *} P<0.001$ versus the vehicle-treated group.

is an important parameter involved in the efficiency of EA [57], we explored the effects of both high- $(100 \mathrm{~Hz})$ and lowfrequency $(2 \mathrm{~Hz}) \mathrm{EA}$ in the present study. Although neither of them had a definite antidepressant-like effect, $2 \mathrm{~Hz}$ EA exerted a remarkable increase in the horizontal movement distance and a better tendency in sucrose preference and immobility time. Thus, we supposed that $2 \mathrm{~Hz}$ EA might be more suitable for combined treatment with a lower dose of citalopram.

As expected, we found that $2 \mathrm{~Hz}$ EA combined with a low dose of citalopram led to significant improvement in the sucrose preference test and FST, showing a greater 
effect on depressive-like behavior than either treatment alone. Similarly, several previous literatures have shown that EA augmented antidepressant-like effects of a tricyclic antidepressant, clomipramine or mianserin, in depression model rats or patients $[54,56,58]$. It has also been reported that application of acupuncture to low-dose fluoxetine-treated depressed patients is as effective as a recommended dose of fluoxetine treatment. However, the mechanisms underlying these combined effects remain unknown.

The delayed onset of SSRIs suggests that their therapeutic effects are mediated beyond a simple enhancement in serotonergic neurotransmission but may be more related to reorganization of neuronal networks [59]. Numerous studies have identified a key role of BDNF in the development and treatment of depression. BDNF needs binding to highaffinity protein kinase receptor family, $\operatorname{TrkB}$, to exert its biological effects, such as neuronal survival, differentiation, neurotransmitter release, and synaptic plasticity. There is a clear evidence that BDNF-TrkB signaling mediates the actions of antidepressants [60]. For instance, chronic SSRI treatment could increase BDNF expression and TrkB receptor activation in rodent hippocampus [25, 61]. Consistent with these findings, the present study demonstrates that chronic administration of $10 \mathrm{mg} / \mathrm{kg}$ citalopram increased both BDNF and TrkB protein levels in rat hippocampus. Moreover, combined treatment with $2 \mathrm{~Hz}$ EA and $5 \mathrm{mg} / \mathrm{kg}$ citalopram, but not either treatment alone, produced similar effects, corresponding to improved depressive-like behavior. However, the relationship between the change of BDNF-TrkB signaling and the antidepressant-like effect of the combined therapy needs further investigation.

In conclusion, our study points out that the combined treatment with EA and a low dose of citalopram could prevent CUS-induced decrease in hippocampal BDNF signaling and lead to greater antidepressant effects than either treatment alone. These findings could provide a new perspective on clinical therapy for depression.

\section{Authors' Contribution}

J. Yang and Y. Pei contributed equally to this work and should be considered co-first authors.

\section{Acknowledgments}

This work was supported by the Grants from National Natural Science Foundation of China (81071102 and 81072858), Beijing Postdoctoral Research Foundation (2011ZZ-10), Training Programme Foundation for the Beijing Municipal Excellent Talents (20081D0301100089), and the National Basic Research Program of China (2011CB504100).

\section{References}

[1] D. Richards, "Prevalence and clinical course of depression: a review," Clinical Psychology Review, vol. 31, no. 7, pp. 1117-1125, 2011.

[2] B. Arroll, S. Macgillivray, S. Ogston et al., "Efficacy and tolerability of tricyclic antidepressants and SSRIs compared with placebo for treatment of depression in primary care: a meta-analysis," Annals of Family Medicine, vol. 3, no. 5, pp. 449-456, 2005.

[3] P. Cassano and M. Fava, "Tolerability issues during long-term treatment with antidepressants," Annals of Clinical Psychiatry, vol. 16, no. 1, pp. 15-25, 2004.

[4] C. M. Dording, D. Mischoulon, T. J. Petersen et al., "The pharmacologic management of SSRI-induced side effects: a survey of psychiatrists," Annals of Clinical Psychiatry, vol. 14, no. 3, pp. 143-147, 2002.

[5] A. Cipriani, T. A. Furukawa, G. Salanti et al., "Comparative efficacy and acceptability of 12 new-generation antidepressants: a multiple-treatments meta-analysis," The Lancet, vol. 373, no. 9665, pp. 746-758, 2009.

[6] C. A. Smith, P. P. Hay, and H. Macpherson, "Acupuncture for depression.," Cochrane Database of Systematic Reviews, no. 1, p. CD004046, 2010.

[7] J. J. B. Allen, R. N. Schnyer, A. S. Chambers, S. K. Hitt, F. A. Moreno, and R. Manber, "Acupuncture for depression: a randomized controlled trial," Journal of Clinical Psychiatry, vol. 67, no. 11, pp. 1665-1673, 2006.

[8] H. Luo, F. Meng, Y. Jia, and X. Zhao, "Clinical research on the therapeutic effect of the electro-acupuncture treatment in patients with depression," Psychiatry and Clinical Neurosciences, vol. 52, pp. S338-S340, 1998.

[9] J. Wu, A. S. Yeung, R. Schnyer, Y. Wang, and D. Mischoulon, "Acupuncture for depression: a review of clinical applications," Canadian Journal of Psychiatry, vol. 57, no. 7, pp. 397-405, 2012.

[10] Z. J. Zhang, R. Ng, S. C. Man et al., "Dense cranial electroacupuncture stimulation for major depressive disorder-a single-blind, randomized, controlled study," PLoS One, vol. 7, no. 1, Article ID e29651, 2012.

[11] Y. Mukaino, J. Park, A. White, and E. Ernst, "The effectiveness of acupuncture for depression-a systematic review of randomised controlled trials," Acupuncture in Medicine, vol. 23, no. 2, pp. 70-76, 2005.

[12] A. F. Thachil, R. Mohan, and D. Bhugra, "The evidence base of complementary and alternative therapies in depression," Journal of Affective Disorders, vol. 97, no. 1-3, pp. 23-35, 2007.

[13] R. J. Leo and J. S. A. Ligot, "A systematic review of randomized controlled trials of acupuncture in the treatment of depression," Journal of Affective Disorders, vol. 97, no. 1-3, pp. 13-22, 2007.

[14] R. Nahas and O. Sheikh, "Complementary and alternative medicine for the treatment of major depressive disorder," Canadian Family Physician, vol. 57, no. 6, pp. 659-663, 2011.

[15] M. M. Poo, "Neurotrophins as synaptic modulators," Nature Reviews Neuroscience, vol. 2, no. 1, pp. 24-32, 2001.

[16] H. Thoenen, "Neurotrophins and neuronal plasticity," Science, vol. 270, no. 5236, pp. 593-598, 1995.

[17] K. Kohara, A. Kitamura, M. Morishima, and T. Tsumoto, "Activity-dependent transfer of brain-derived neurotrophic factor to postsynaptic neurons," Science, vol. 291, no. 5512, pp. 24192423, 2001.

[18] M. A. H. Rot, S. J. Mathew, and D. S. Charney, "Neurobiological mechanisms in major depressive disorder," Canadian Medical Association Journal, vol. 180, no. 3, pp. 305-313, 2009.

[19] E. Castren, "Is mood chemistry?" Nature Reviews Neuroscience, vol. 6, no. 3, pp. 241-246, 2005.

[20] E. Castren, V. Voikar, and T. Rantamaki, "Role of neurotrophic factors in depression," Current Opinion in Pharmacology, vol. 7, no. 1, pp. 18-21, 2007. 
[21] H. Yu and Z. Y. Chen, "The role of BDNF in depression on the basis of its location in the neural circuitry," Acta Pharmacologica Sinica, vol. 32, no. 1, pp. 3-11, 2011.

[22] B. Chen, D. Dowlatshahi, G. M. MacQueen, J. F. Wang, and L. T. Young, "Increased hippocampal BDNF immunoreactivity in subjects treated with antidepressant medication," Biological Psychiatry, vol. 50, no. 4, pp. 260-265, 2001.

[23] F. Karege, G. Vaudan, M. Schwald, N. Perroud, and R. La Harpe, "Neurotrophin levels in postmortem brains of suicide victims and the effects of antemortem diagnosis and psychotropic drugs," Molecular Brain Research, vol. 136, no. 1-2, pp. 29-37, 2005.

[24] Y. Dwivedi, H. S. Rizavi, R. R. Conley, R. C. Roberts, C. A. Tamminga, and G. N. Pandey, "Altered gene expression of brainderived neurotrophic factor and receptor tyrosine kinase B in postmortem brain of suicide subjects," Archives of General Psychiatry, vol. 60, no. 8, pp. 804-815, 2003.

[25] M. Nibuya, S. Morinobu, and R. S. Duman, "Regulation of $\mathrm{BDNF}$ and trkB mRNA in rat brain by chronic electroconvulsive seizure and antidepressant drug treatments," Journal of Neuroscience, vol. 15, no. 11, pp. 7539-7547, 1995.

[26] A. M. Rasmusson, L. Shi, and R. Duman, "Downregulation of BDNF mRNA in the hippocampal dentate gyrus after reexposure to cues previously associated with footshock," Neuropsychopharmacology, vol. 27, no. 2, pp. 133-142, 2002.

[27] H. Xu, Z. Chen, J. He et al., "Synergetic effects of quetiapine and venlafaxine in preventing the chronic restraint stressinduced decrease in cell proliferation and BDNF expression in rat hippocampus," Hippocampus, vol. 16, no. 6, pp. 551-559, 2006.

[28] Y. Zhang, F. Gu, J. Chen, and W. Dong, "Chronic antidepressant administration alleviates frontal and hippocampal BDNF deficits in CUMS rat," Brain Research, vol. 1366, no. C, pp. 141148,2010

[29] I. K. Hwang, J. Y. Chung, D. Y. Yoo et al., "Effects of electroacupuncture at zusanli and baihui on brain-derived neurotrophic factor and cyclic AMP response element-binding protein in the hippocampal dentate gyrus," Journal of Veterinary Medical Science, vol. 72, no. 11, pp. 1431-1436, 2010.

[30] B. Lee, B. J. Sur, S. Kwon et al., "Acupuncture stimulation alleviates corticosterone-induced impairments of spatial memory and cholinergic neurons in rats," Evidence-Based Complementary and Alternative Medicine, vol. 2012, Article ID 670536, 14 pages, 2012.

[31] S. J. Yun, H. J. Park, M. J. Yeom, D. H. Hahm, H. J. Lee, and E. H. Lee, "Effect of electroacupuncture on the stress-induced changes in brain-derived neurotrophic factor expression in rat hippocampus," Neuroscience Letters, vol. 318, no. 2, pp. 85-88, 2002.

[32] R. J. Katz, "Animal models and human depressive disorders," Neuroscience and Biobehavioral Reviews, vol. 5, no. 2, pp. 231246, 1981.

[33] P. Willner, A. Towell, D. Sampson, S. Sophokleous, and R. Muscat, "Reduction of sucrose preference by chronic unpredictable mild stress, and its restoration by a tricyclic antidepressant," Psychopharmacology, vol. 93, no. 3, pp. 358-364, 1987.

[34] M. Banasr, G. W. Valentine, X. Y. Li, S. L. Gourley, J. R. Taylor, and R. S. Duman, "Chronic unpredictable stress decreases cell proliferation in the cerebral cortex of the adult rat," Biological Psychiatry, vol. 62, no. 5, pp. 496-504, 2007.

[35] K. R. Luo, C. J. Hong, Y. J. Liou, S. J. Hou, Y. H. Huang, and S. J. Tsai, "Differential regulation of neurotrophin S100B and
BDNF in two rat models of depression," Progress in NeuroPsychopharmacology and Biological Psychiatry, vol. 34, no. 8, pp. 1433-1439, 2010.

[36] R. D. Porsolt, G. Anton, N. Blavet, and M. Jalfre, "Behavioural despair in rats: a new model sensitive to antidepressant treatments," European Journal of Pharmacology, vol. 47, no. 4, pp. 379-391, 1978.

[37] Y. Shirayama, A. C. H. Chen, S. Nakagawa, D. S. Russell, and R. S. Duman, "Brain-derived neurotrophic factor produces antidepressant effects in behavioral models of depression," Journal of Neuroscience, vol. 22, no. 8, pp. 3251-3261, 2002.

[38] R. J. Katz, "Animal model of depression: pharmacological sensitivity of a hedonic deficit," Pharmacology Biochemistry and Behavior, vol. 16, no. 6, pp. 965-968, 1982.

[39] P. Willner, "Chronic mild stress (CMS) revisited: consistency and behavioural- neurobiological concordance in the effects of CMS," Neuropsychobiology, vol. 52, no. 2, pp. 90-110, 2005.

[40] P. Willner, "Validity, reliability and utility of the chronic mild stress model of depression: a 10-year review and evaluation," Psychopharmacology, vol. 134, no. 4, pp. 319-329, 1997.

[41] X. Y. Lu, C. S. Kim, A. Frazer, and W. Zhang, "Leptin: a potential novel antidepressant," Proceedings of the National Academy of Sciences of the United States of America, vol. 103, no. 5, pp. 1593$1598,2006$.

[42] H. Xu, H. Qing, W. Lu et al., "Quetiapine attenuates the immobilization stress-induced decrease of brain-derived neurotrophic factor expression in rat hippocampus," Neuroscience Letters, vol. 321, no. 1-2, pp. 65-68, 2002.

[43] S. C. Dulawa, K. A. Holick, B. Gundersen, and R. Hen, "Effects of chronic fluoxetine in animal models of anxiety and depression," Neuropsychopharmacology, vol. 29, no. 7, pp. 13211330, 2004.

[44] C. López-Rubalcava and I. Lucki, "Strain differences in the behavioral effects of antidepressant drugs in the rat forced swimming test," Neuropsychopharmacology, vol. 22, no. 2, pp. 191-199, 2000.

[45] C. Sánchez and E. Meier, "Behavioral profiles of SSRIs in animal models of depression, anxiety and aggression. Are they all alike?" Psychopharmacology, vol. 129, no. 3, pp. 197-205, 1997.

[46] G. Wegener, Z. Bandpey, I. L. Heiberg, A. Mørk, and R. Rosenberg, "Increased extracellular serotonin level in rat hippocampus induced by chronic citalopram is augmented by subchronic lithium: neurochemical and behavioural studies in the rat," Psychopharmacology, vol. 166, no. 2, pp. 188-194, 2003.

[47] M. Tõnissaar, T. Mällo, M. Eller, R. Häidkind, K. Kõiv, and J. Harro, "Rat behavior after chronic variable stress and partial lesioning of 5-HT-ergic neurotransmission: effects of citalopram," Progress in Neuro-Psychopharmacology and Biological Psychiatry, vol. 32, no. 1, pp. 164-177, 2008.

[48] M. Kuźmider, J. Solich, P. Pałach, and M. DziedzickaWasylewska, "Effect of citalopram in the modified forced swim test in rats," Pharmacological Reports, vol. 59, no. 6, pp. 785-788, 2007.

[49] E. Takahashi, M. Katayama, K. Niimi, and C. Itakura, "Additive subthreshold dose effects of cannabinoid CB1 receptor antagonist and selective serotonin reuptake inhibitor in antidepressant behavioral tests," European Journal of Pharmacology, vol. 589, no. 1-3, pp. 149-156, 2008.

[50] Y. C. Chen, Q. R. Tan, W. Dang et al., "The effect of citalopram on chronic stress-induced depressive-like behavior in rats through GSK3beta/beta-catenin activation in the medial 
prefrontal cortex," Brain Research Bulletin, vol. 88, no. 4, pp. 338-344, 2012.

[51] C. P. France, J. X. Li, W. A. Owens, W. Koek, G. M. Toney, and L. C. Daws, "Reduced effectiveness of escitalopram in the forced swimming test is associated with increased serotonin clearance rate in food-restricted rats," International Journal of Neuropsychopharmacology, vol. 12, no. 6, pp. 731-736, 2009.

[52] R. Rygula, N. Abumaria, G. Flügge et al., "Citalopram counteracts depressive-like symptoms evoked by chronic social stress in rats," Behavioural Pharmacology, vol. 17, no. 1, pp. 19-29, 2006.

[53] S. H. Wang, Z. J. Zhang, Y. J. Guo, H. Zhou, G. J. Teng, and B. A. Chen, "Anhedonia and activity deficits in rats: impact of poststroke depression," Journal of Psychopharmacology, vol. 23, no. 3, pp. 295-304, 2009.

[54] J. Yu, Q. Liu, Y. Q. Wang et al., "Electroacupuncture combined with clomipramine enhances antidepressant effect in rodents," Neuroscience Letters, vol. 421, no. 1, pp. 5-9, 2007.

[55] J. H. Liu, Z. F. Wu, J. Sun, L. Jiang, S. Jiang, and W. B. Fu, "Role of AC-cAMP-PKA cascade in antidepressant action of electroacupuncture treatment in rats," Evidence-Based Complementary and Alternative Medicine, vol. 2012, Article ID 932414, 7 pages, 2012.

[56] J. Yu, X. Y. Li, X. D. Cao, and G. C. Wu, "Sucrose preference is restored by electro-acupuncture combined with chlorimipramine in the depression-model rats," Acupuncture and Electro-Therapeutics Research, vol. 31, no. 3-4, pp. 223-232, 2006.

[57] X. B. Liang, Y. Luo, X. Y. Liu et al., "Electro-acupuncture improves behavior and upregulates GDNF mRNA in MFB transected rats," NeuroReport, vol. 14, no. 8, pp. 1177-1181, 2003.

[58] J. Röschke, C. Wolf, M. J. Müller et al., "The benefit from whole body acupuncture in major depression," Journal of Affective Disorders, vol. 57, no. 1-3, pp. 73-81, 2000.

[59] E. Castrén and T. Rantamäki, "The role of BDNF and its receptors in depression and antidepressant drug action: reactivation of developmental plasticity," Developmental Neurobiology, vol. 70, no. 5, pp. 289-297, 2010.

[60] J. H. Krystal, D. F. Tolin, G. Sanacora et al., "Neuroplasticity as a target for the pharmacotherapy of anxiety disorders, mood disorders, and schizophrenia," Drug Discovery Today, vol. 14, no. 13-14, pp. 690-697, 2009.

[61] T. Saarelainen, P. Hendolin, G. Lucas et al., "Activation of the TrkB neurotrophin receptor is induced by antidepressant drugs and is required for antidepressant-induced behavioral effects," Journal of Neuroscience, vol. 23, no. 1, pp. 349-357, 2003. 


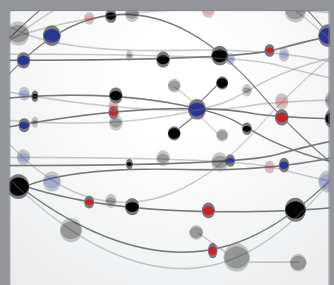

The Scientific World Journal
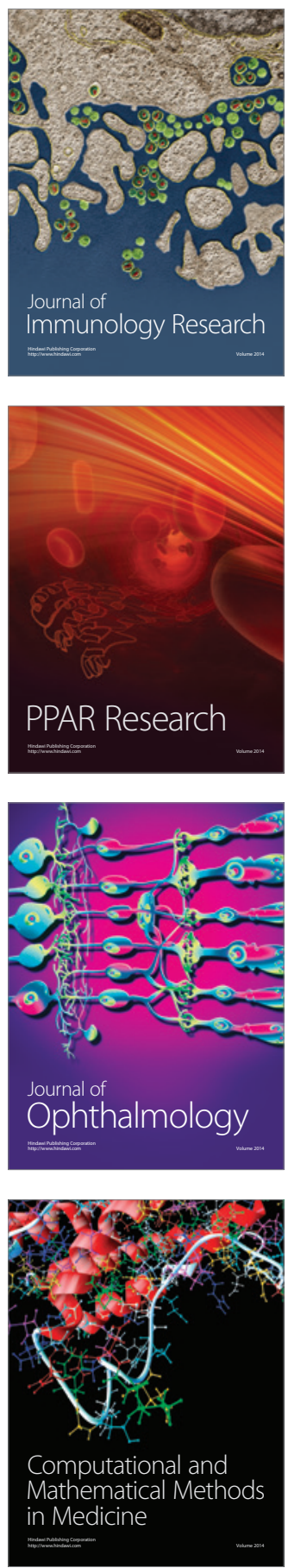

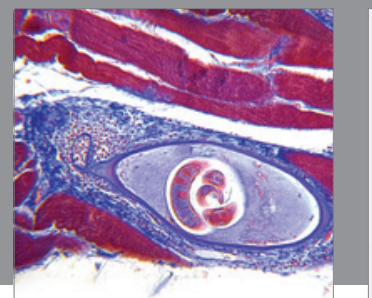

Gastroenterology

Research and Practice
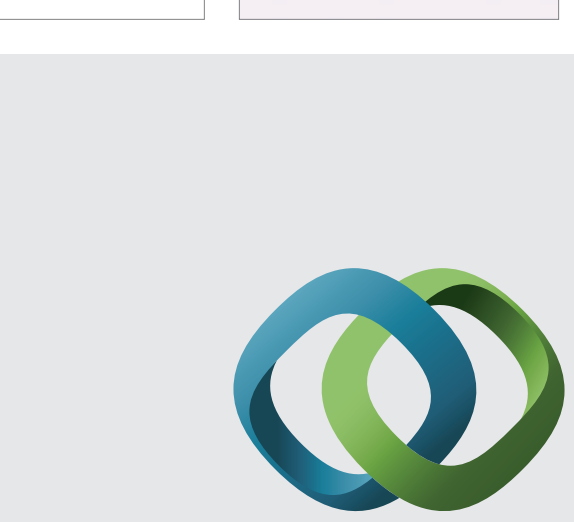

\section{Hindawi}

Submit your manuscripts at

http://www.hindawi.com
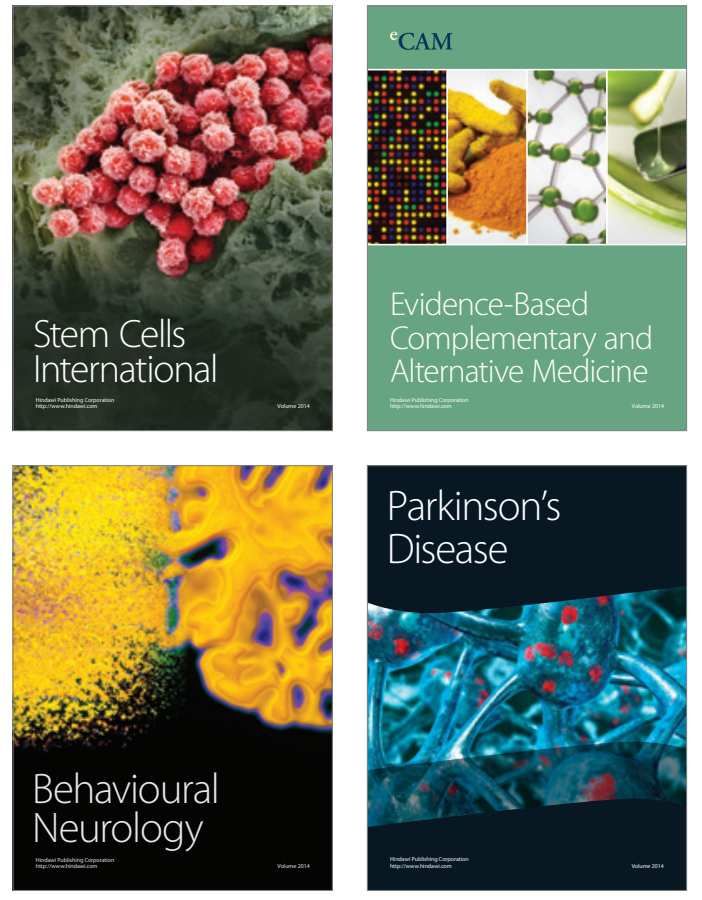
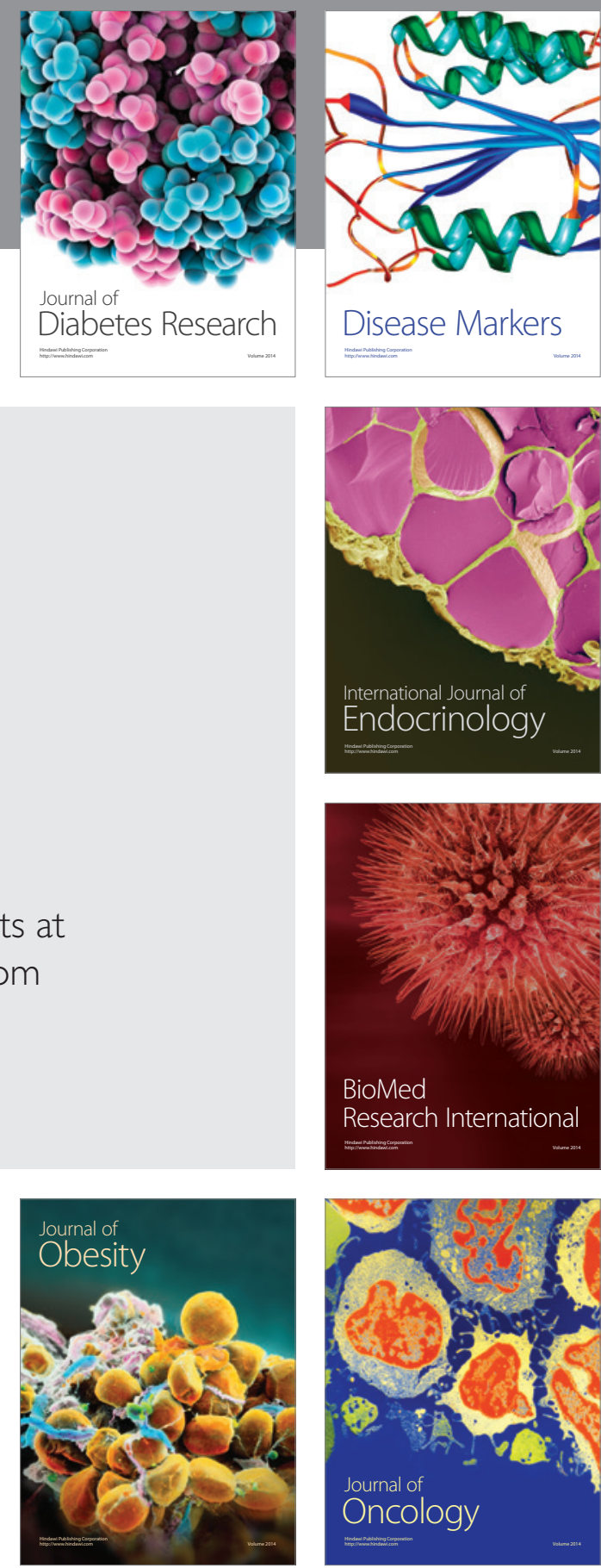

Disease Markers
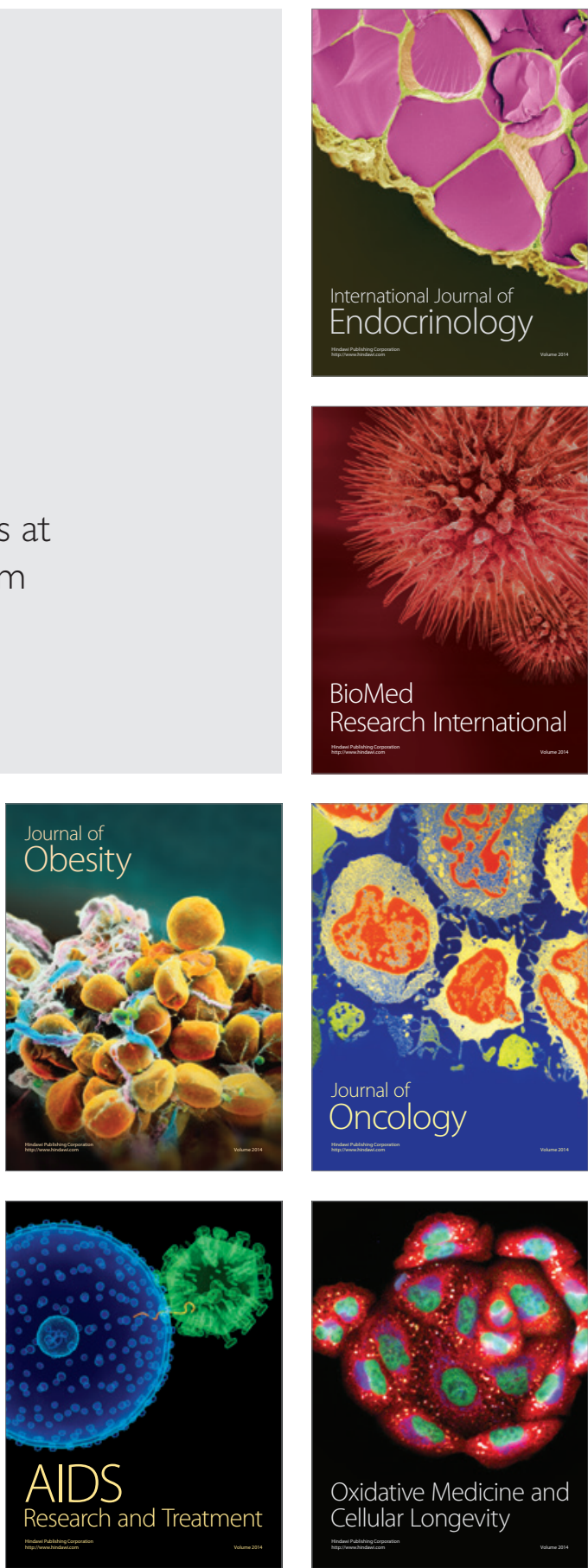